University of Wollongong

Research Online

Faculty of Engineering and Information

Faculty of Engineering and Information

Sciences - Papers: Part A

Sciences

$1-1-2013$

\title{
A molecular dynamics simulation of fracture in nanocrystalline copper
}

\author{
Linqing Pei \\ University of Wollongong, Ip115@uowmail.edu.au \\ Cheng Lu \\ University of Wollongong, chenglu@uow.edu.au \\ Kiet Tieu \\ University of Wollongong, ktieu@uow.edu.au \\ Hongtao Zhu \\ University of Wollongong, hongtao@uow.edu.au \\ Xing Zhao \\ University of Wollongong, xz920@uowmail.edu.au
}

See next page for additional authors

Follow this and additional works at: https://ro.uow.edu.au/eispapers

Part of the Engineering Commons, and the Science and Technology Studies Commons

Research Online is the open access institutional repository for the University of Wollongong. For further information contact the UOW Library: research-pubs@uow.edu.au 


\title{
A molecular dynamics simulation of fracture in nanocrystalline copper
}

\author{
Abstract \\ A large-scale molecular dynamics simulation was used to investigate the propagation of cracks in three \\ dimensional samples of nanocrystalline copper, with average grain sizes ranging from 5.34 to $14.8 \mathrm{~nm}$ \\ and temperatures ranging from $1 \mathrm{~K}$ to $500 \mathrm{~K}$. It was shown that intragranular fracture can proceed inside \\ the grain at low temperature, and plastic deformation around the tip of the crack is accommodated by \\ dislocation nucleation/emission; indeed, both fully extended dislocation and deformation twinning were \\ visible around the tip of the crack during fracture. In addition, due to a higher concentration of stress in \\ front of the crack at a relative lower temperature, it was found that twinning deformation is easier to \\ nucleate from the tip of the crack. These results also showed that the decreasing grain size below a \\ critical value exhibits a reverse Hall-Petch relationship due to the enhancing grain boundary mediation, \\ and high temperature is better for propagating ductile cracks. () (2013) Trans Tech Publications, \\ Switzerland. \\ Keywords \\ nanocrystalline, copper, fracture, molecular, simulation, dynamics \\ Disciplines \\ Engineering I Science and Technology Studies \\ Publication Details \\ Pei, L., Lu, C., Tieu, K., Zhu, H., Zhao, X., Cheng, K. \& Zhang, L. (2013). A molecular dynamics simulation of \\ fracture in nanocrystalline copper. Journal of Nano Research, 23 50-56.

\section{Authors} \\ Linqing Pei, Cheng Lu, Kiet Tieu, Hongtao Zhu, Xing Zhao, Kuiyu Cheng, and Liang Zhang
}

This journal article is available at Research Online: https://ro.uow.edu.au/eispapers/1309 


\title{
A Molecular Dynamics Simulation of Fracture in Nanocrystalline Copper
}

\author{
Linqing Pei ${ }^{\mathrm{a}}$, Cheng $\mathrm{Lu}^{\mathrm{b}}$, Kiet Tieu ${ }^{\mathrm{c}}$, Hongtao Zhu ${ }^{\mathrm{d}}$, Xing Zhao ${ }^{\mathrm{e}}$, Kuiyu Cheng ${ }^{f}$, \\ Liang Zhang ${ }^{g}$
}

School of Mechanical, Materials and Mechatronic Engineering

University of Wollongong, Wollongong, NSW 2522, Australia

alp115@uowmail.edu.au, bchenglu@uow.edu.au (corresponding author)

[Submitted: November 3, 2012;revised: March 12, 2013; accepted: April 8, 2013]

\begin{abstract}
Keywords: Crack propagation, Intragranular fracture, MD simulation, Nanocrystalline copper, Deformation twinning.
\end{abstract}

\begin{abstract}
A large-scale molecular dynamics simulation was used to investigate the propagation of cracks in three dimensional samples of nanocrystalline copper, with average grain sizes ranging from 5.34 to $14.8 \mathrm{~nm}$ and temperatures ranging from $1 \mathrm{~K}$ to $500 \mathrm{~K}$. It was shown that intragranular fracture can proceed inside the grain at low temperature, and plastic deformation around the tip of the crack is accommodated by dislocation nucleation/emission; indeed, both fully extended dislocation and deformation twinning were visible around the tip of the crack during fracture. In addition, due to a higher concentration of stress in front of the crack at a relative lower temperature, it was found that twinning deformation is easier to nucleate from the tip of the crack. These results also showed that the decreasing grain size below a critical value exhibits a reverse Hall-Petch relationship due to the enhancing grain boundary mediation, and high temperature is better for propagating ductile cracks.
\end{abstract}

\section{Introduction}

Fracture generally includes two categories of failure: brittle and ductile. These types of behaviour mainly depend on two major competing modes: dislocation nucleation vs. the energy required to create new surfaces around a crack for conventional crystals [1, 2]. However, when the grains are reduced in size to some small nano-scales, the dislocation activity may be suppressed by the grain boundary (GB) domination, at which stage the brittle vs. ductile behaviour is decided by two competing mechanisms: (1) GB-induced plastic deformation (crack blunting) and (2) GB decohesion due to the evolution of voids (crack propagation) $[3,4]$.

In recent years large scale atomic level simulations have provided an insight into the atomic scale processes of fracture dynamics and making considerable progress in understanding the fundamentals of fracture. Buehler studied the hyper-elasticity governing the dynamic fracture in brittle materials [5]. Hai $[6,7]$ et al found deformation twinning occurring at the tips of cracks in aluminum at certain combinations of loading and orientation. Warner[8] successfully revealed a crack-tip twinning transition at a short time to full dislocation at a long time, in fcc single crystals. The embrittlement of hydrogen in single crystal Ni was analysed by enhancing the decohesion for fracture[9]. Farkas [10-13] developed a method of embedding atoms to observe intergranular facture along the grain boundaries. This was accomplished by coalescing the nano-voids ahead of the crack in polycrystal $\mathrm{Ni}$ and Fe, where the plasticity around the tip of the crack was contained by the dislocation/twinning activities. They also discussed whether the grain size and impurities would improve the ductility of nanocrystalline metals. Furthermore, in the literatures $[14,15]$ both intragranular and intergranular fracture were observed. Other people found that a material should not be simply classified as intrinsically brittle or ductile [16] because under some specific conditions of mechanical load, the nanocrystalline materials may be shown to exhibit either brittle or ductile fracture behaviour. The sudden transition from brittle to ductile behaviour of a silicon single crystal has been captured by Sen 
[17]. He revealed that this change can be completed within a $10 \mathrm{~K}$ temperature interval due to the generation of a crack-tip ledge for dislocation emission. Moreover, other brittle to ductile transitions were also observed in simulations $[18,19]$ of single crystal Fe. As mentioned above, previous atomic level simulations have mainly focused on the study of fracture in metals like $\mathrm{Al}, \mathrm{Ni}, \mathrm{Si}$, and $\mathrm{Fe}$, however very little work has been done on the initial formation of cracks in copper polycrystals, so it is of great interest to investigate the fracture properties of $\mathrm{Cu}$ poly-crystal.

In this study, we introduced a pre-existing semi-infinite crack in the middle of a $\mathrm{Cu}$ polycrystal, and then used a molecular dynamics simulation to investigate the process of atomic configuration at the tip of the crack by considering the various temperatures and grain sizes.

\section{Modeling}

The copper polycrystal sample was generated using the Voronoi construction with random seeds and random crystallographic orientations, and its geometry is $216.9 * 216.9 * 216.9$ angstrom in $\mathrm{X}, \mathrm{Y}$ and Z directions, respectively. An initially semi-infinite crack was inserted into the left part of the sample with $100 * 14.46 * 216.9$ angstrom (in X, Y, Z direction). Different mean grain diameters $(5.34,7.31$, $10.68,12.34,14.8 \mathrm{~nm})$ and temperatures $(1,100,300,500 \mathrm{~K})$ were simulated to investigate the behaviour of the fracture for polycrystal. An EAM (Embedded atom method) interatomic potential [20] which is based on the ab initio method was adopted and all simulations were performed using a Nose-Hoover thermostat and a Parinello-Rahman barostat [21]. A multiple time algorithm was used with a time step of 1 femtosecond. Periodic boundary conditions were imposed on all three directions. After the polycrystal model was constructed, the sample was relaxed [13] to free enthalpy minimum at given temperatures and 0 bar external stress for 30000 femtoseconds before tensile deformation. Then a relatively large constant rate of $1 * 10^{9} \mathrm{~s}^{-1}$ was applied along the $\mathrm{Y}$ axis until it reached a stretched strain of $20 \%$. In order to discern the perfect atoms and imperfect atoms like the grain boundary structure or other defects, the atoms were given different colours according to the local crystalline order by the common neighbours analysis [22]: deep blue represents fcc atoms, light blue represents hcp atoms, and red represents not-12 coordinated atoms. A single light blue line stands for a twinning, two adjacent hep lines stand for an intrinsic stacking fault, and two hep lines with an fcc line between them stand for an extrinsic stacking fault. This method provides a very efficient way of identifying perfect fcc atoms and other twinning/dislocation atoms or disorder atoms.

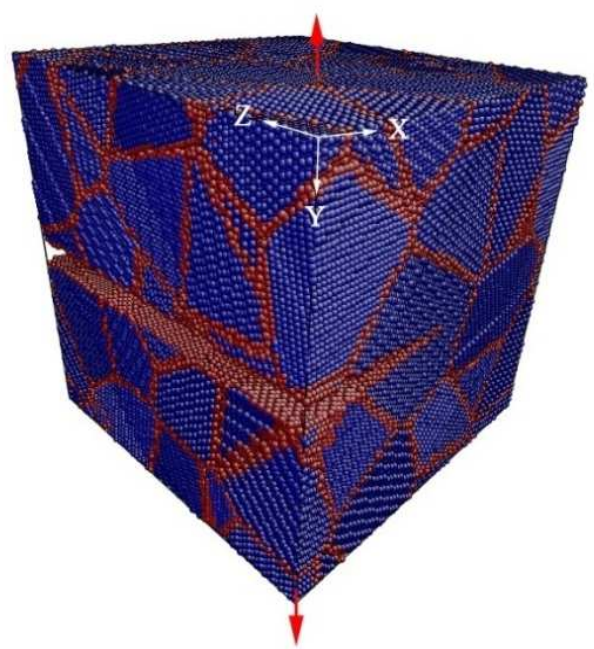

Fig. 1 Schematic of mode I fracture model with semi-infinite crack. The red arrows represent the mode I loading applied direction. 


\section{Results and Discussion}

It is well known that the grain boundary and crack tip can serve as a site for nucleation and a sink for dislocations [10,11], and the behaviour of cracks is widely recognised, depending on the competition between the cleavage decohesion and dislocation nucleation/GB-induced deformation [6]. Fig. 2 shows the presence of partial dislocation and a fully extended dislocation around the tip of the crack. In Fig. 2 (a) is the initiated atomic model, in Fig. 2 (b), as the applied strain is increased. The stress also increases and then concentrates itself at the tip of the crack due to geometric discontinuity, but once the stress reaches its threshold value for dislocation emission, the crack blunts with a series of partial dislocations on the $\{111\}$ slip plane being emitted from the tip of the crack, meanwhile, the occurrence of crack bluntness can itself affect the dislocation emission and crack propagation [16, 23]. In Fig. 2 (c), it can be seen that other partial dislocations are emitted from the tip of the crack and the triple junction of the grain boundaries, and travel through the grain to the opposite grain boundary. These dislocations emitted from the crack or triple junctions of grain boundaries effectively blunt the crack and suppress its growth [24] In Fig. 2 (d) and (e), once the leading partial dislocation is being nucleated in the $\{111\}$ slip plane, the second partial dislocation can be nucleated in the same plane in the region of interior grain, which means that a full homogeneous dislocation is nucleated inside the grain and is finally arrested on the other side of the grain boundary. The formation of partial dislocations or fully extended dislocations can be explained in terms of the generalised planar fault (GPF) energy curve [25]. In comparison with other high stacking energy materials like aluminum, the energy barrier that must be overcome to create a trailing partial is very high in copper, so it is more frequent to see the formation of partial dislocation in copper. In Fig. 2 (f), a further sharp crack deviating from the original crack plane to another plane is going forward due to the low temperature, in the vicinity of the crack tip, and a three-layer microtwin is generated.

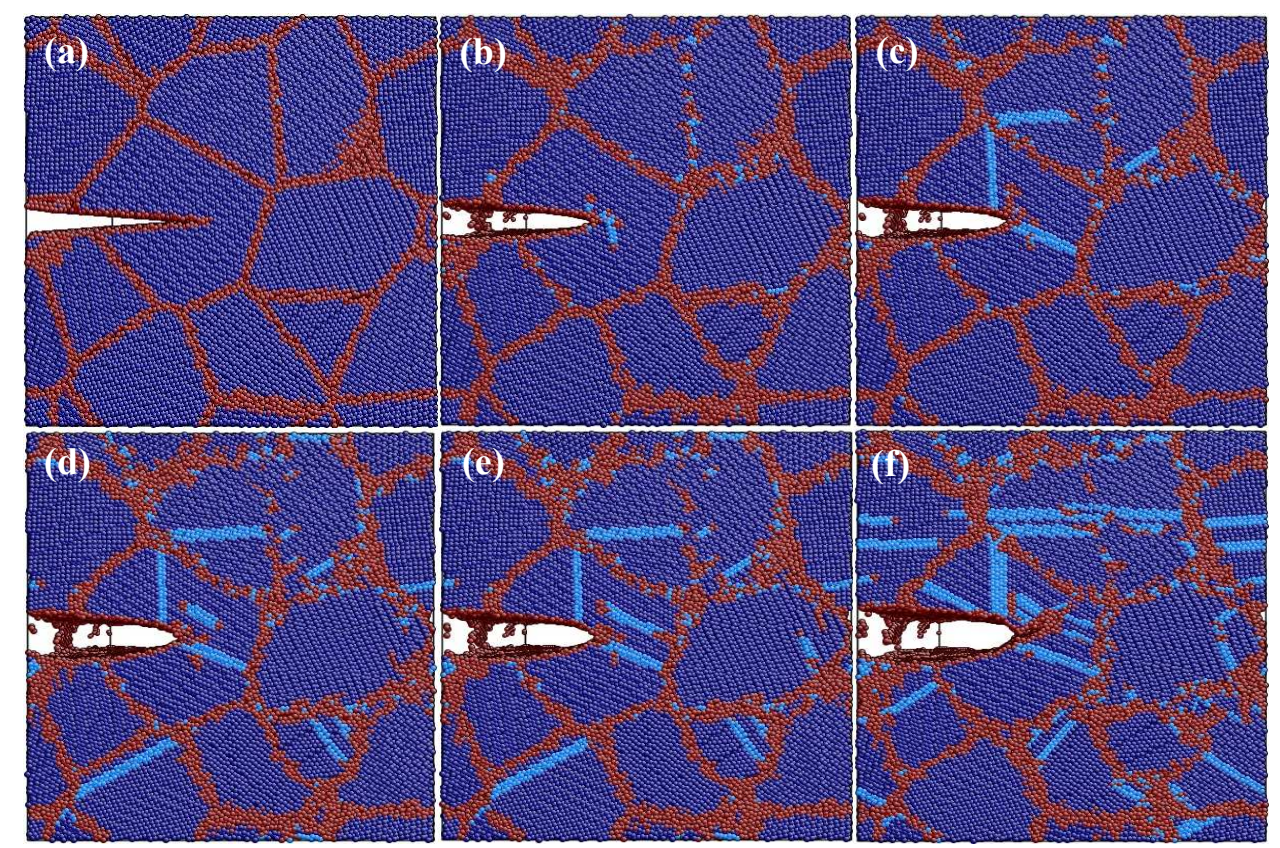

Fig. 2 A sequence of events (a) (b) (c) (d) (e) (f) for a section of the sample at $1 \mathrm{k}$ with grain size of $7.31 \mathrm{~nm}$ showing the emission of leading and trailing partial dislocation from the crack tip or the neighbouring grain boundaries.

Fig. 3 shows the formation of a twinning by the emission of consecutive leading Shockley partials on neighboring $\{111\}$ slip planes. The partial dislocations are all travelling through the entire grain to the opposite grain boundary which allows a twinning lamellae to form and extend itself from the tip of the crack to the grain boundary. This mechanism is similar to the heterogeneous formation of twinning found in the previous tensile deformation for Al [26], except that the twinning lamellae are now affected by the tip of the crack. Twinning nucleation can be understood as an energetic point 
where the ratio of unstable twin fault energy of unstable stacking fault energy is close to 1 for $\mathrm{Cu}$ [25], which results in a energetically preferable twinning formation in copper. In other words, this process is mainly dependent on competition between the emission of a trailing partial in the same plane or in the adjacent slip plane, after the leading partial dislocation has been nucleated. It was found that the formation of deformation twinning is strongly correlated with the temperature applied and orientation of the front of the crack. It is hoped that the nucleated dislocation or twinning in fcc structure, will increase the fracture toughness because it is easy to see in Fig. 3 that the crack blunts gradually after the nucleation of twinning/dislocation.
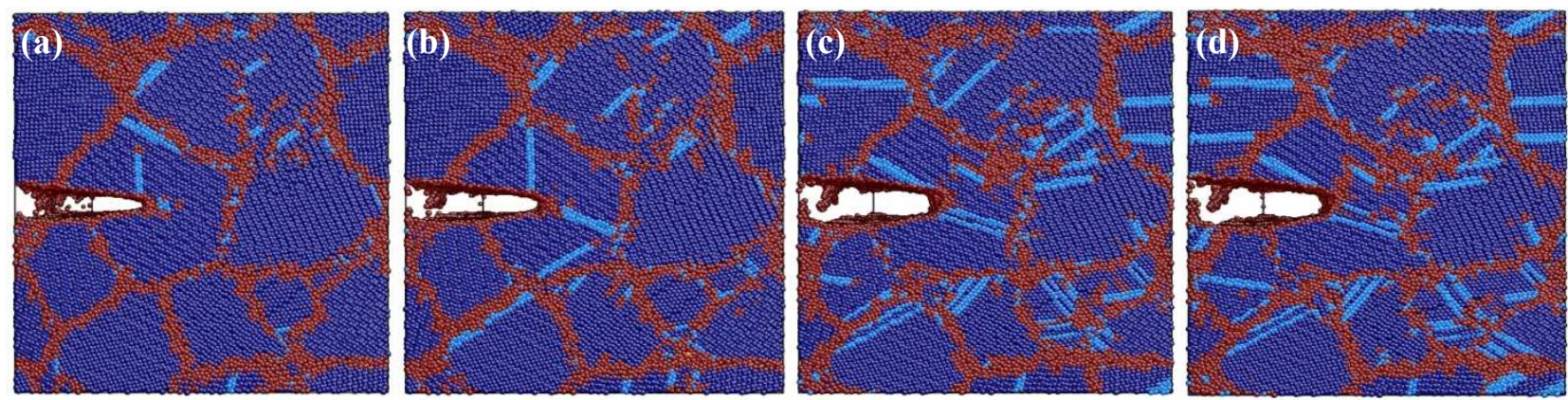

Fig. 3 A sequence of snapshots (a) (b) (c) (d) for twinning formation at $100 \mathrm{k}$ for grain size of $7.31 \mathrm{~nm}$.

Since we know that low temperature favours brittle fracture, Fig. 4 (a)-(d) contains a comparison of 4 groups of temperatures, where the results show that at low temperatures, cracks propagates in a more brittle manner, but as the temperature increases, the system will exhibit more crack blunting. Apart from this, our simulations also show that deformation twinning is affected by the effects of temperature such that at low temperatures $(1 \mathrm{~K}, 100 \mathrm{~K})$, twinning is more likely to be nucleated because of the larger concentration of stress generated around the tip of the crack at lower temperatures. Fig. 5 (a) shows the engineering stress vs. strain curves under varied temperatures, where a low temperature presents a bigger flow stress trend. With coarser grains, the yield stress increases with a decreasing mean grain size because it is believed to obey the Hall-Petch relationship. However, the situation will be reversed (called reverse Hall-Petch) when the mean grain size reaches the critical nanocrystalline scale (average diameter of about $12.34 \mathrm{~nm}$, the strongest size), as seen in Fig. 5 (b), because the effect of mean grain size on the mechanical properties obeying the reverse Hall-Petch relation is validated. This is because the increasing volume of grain boundaries in the model raises the possibility of porosity at a very small grain size, which suppresses the dislocation pile-ups and motion more while the now enhanced grain boundary activities such as grain boundary rotation, grain boundary sliding and diffusion creep [27-29], leads to a characteristic reverse Hall-Petch relationship.
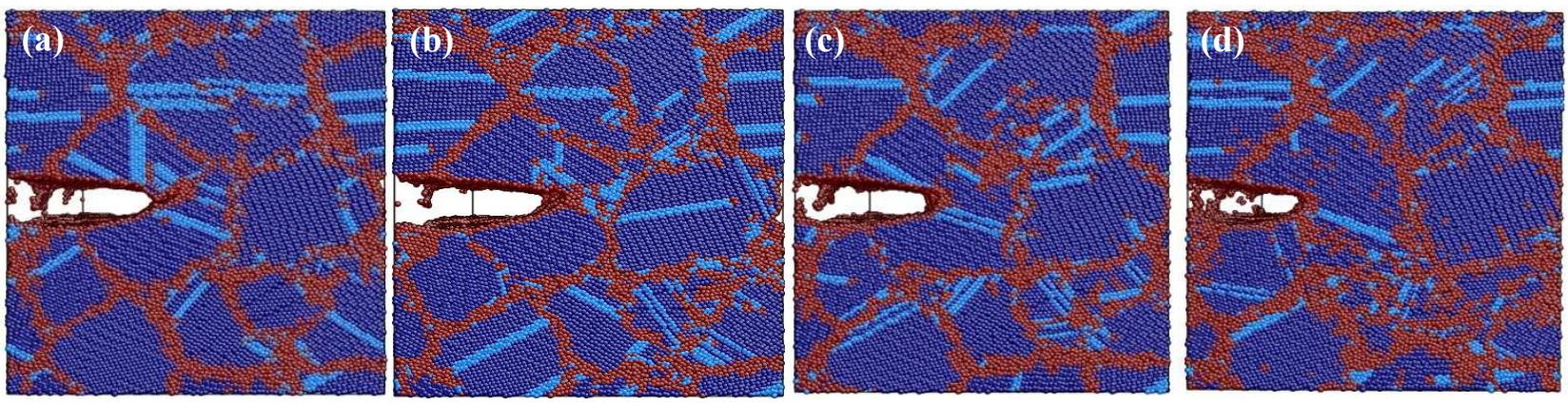

Fig. 4 Crack propagation under different temperatures at same strain deformation for grain size of $7.31 \mathrm{~nm}$ : (a) $1 \mathrm{k}$, (b) $100 \mathrm{k}$, (c) $300 \mathrm{k}$, (d) $500 \mathrm{k}$. 
In every case we can see that all the fractures show a very pronounced blunting behaviour for the fracture of copper polycrystal, which is consistent with most fcc structures exhibiting a blunt fracture. This in unlike the bcc structure (like Fe) where at low temperature, the fracture behaves in a more dominant brittle manner because the locally concentrated load which is required for brittle propagating is normally easier than partial/full dislocation emission in front of the crack, although in some conditions brittle-ductile transition from a low temperature to a higher temperature will occur. Although there was dominant blunting fracture behaviour in our simulations, the decreasing grain size can still promote a slight ductility, owing to the improving capability of the grain boundary accommodation.

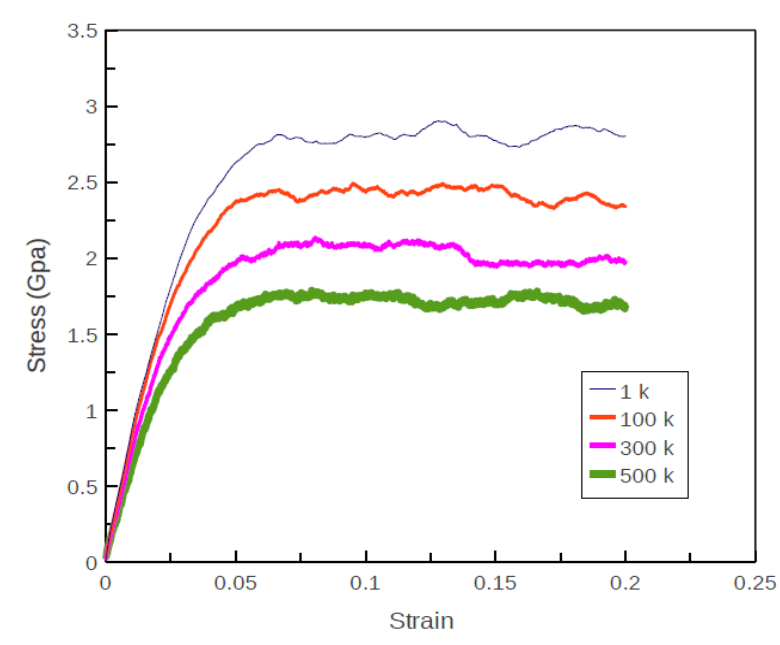

(a)

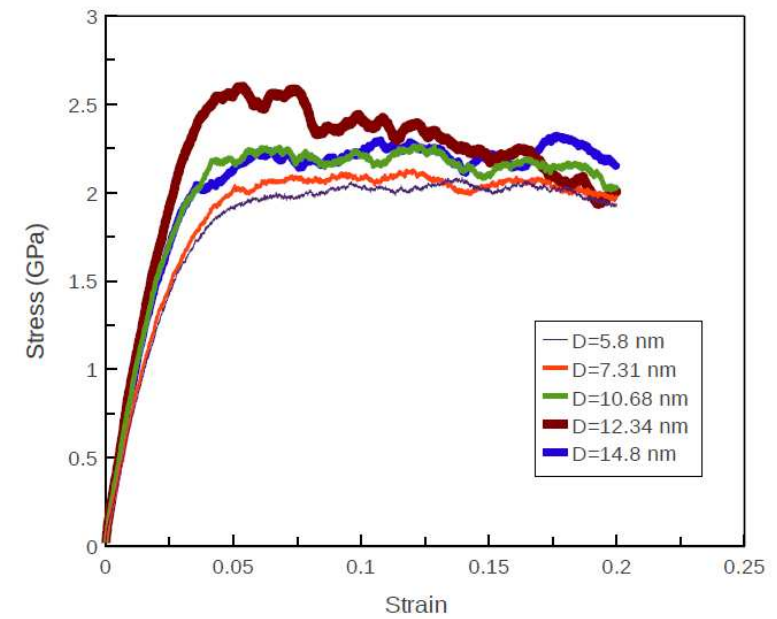

(b)

Fig. 5 (a) Stress vs strain curves with varied temperature for semi-infinite crack model with average grain size of $7.31 \mathrm{~nm}$. (b) Stress-strain curves for polycrystal copper with different grain sizes D at $300 \mathrm{k}$.

It is worth noting that in our simulations, only intragranular fracture rather than intergranular fracture[11, 15] was visible along the grain boundaries. This discrepancy may be ascribed to the fixed boundary conditions, the initial created position of the tip of the crack, the orientations of the grain boundary, and the grain boundary volume ratio in front of the crack. In those models a crack was inserted inside the grain and far away from the nearest grain boundary, which may result in a more favourable intragranular fracture rather than an intergranular fracture. In addition, the distribution and orientation of the grain boundary, and the percentage of grain boundary volume also influenced the form of fracture behaviour $[3,14]$ No crack necking occurred in the simulations because of the periodic boundary conditions applied to the small scale samples. This raises an interesting question of how to improve the brittle/ductile fracture in fcc and bec structures (polycrystal), or even finding a brittle-ductile transition using molecular dynamics simulation which has already been confirmed in in situ experiments [30,31]. Moreover the following deformation parameters may be also be considered: low temperature, shape of the created crack, crack and sample geometry, inclusions, average distribution of grain size, orientation of the grain boundary, the ratio of the grain boundary volume in the whole model, favourable oriented misorientation of grain atoms, fixed boundary conditions, and the mode of the force applied, and its value. In these circumstances we may find a replicated characteristic found in situ experiments using the MD method. We must also keep in mind that owing to the intrinsic limitations in the time and length of molecular dynamics simulation, plus the difficulties in preparing high quality samples for experiments [4], an accurate description of the behaviour of fracture at the nano-scale is still far from being developed. 


\section{Conclusions}

A molecular dynamics simulation was used to investigate the propagation of fracture in copper polycrystal using EAM potential. The models indicated that to accommodate the propagation of cracks, partial dislocation/twinning is nucleated from the grain boundary triple junctions, the interior of the grain boundary, or the tip of the crack, and this dislocation activity can be understood by virtue of the GPF curves. Intragranular fracture combined with deformation twinning at the tip of the crack was observed, especially at low temperature. It was shown that low temperature benefits brittle fracture and the formation of twinning, but when the grains decrease in size to an extreme value at the nano-scale, the mechanical properties are obeying the reverse Hall-Petch relationship where the stress increases as the grains decrease in size due to the large fraction of grain boundaries.

\section{Acknowledgments}

We would like to thank the Lammps group members and Ju Li for providing the analytical tool for MD pre-processing and visualization.

\section{References}

[1] J.R. Rice, R. Thomson, Ductile vs Brittle Behaviour of Crystals, Phil Mag, 29 (1974) 73-97.

[2] J.R. Rice, Dislocation nucleation from a crack tip: An analysis based on the Peierls concept, Journal of the Mechanics and Physics of Solids, 40 (1992) 239-271.

[3] F. Yang, W. Yang, Brittle versus ductile transition of nanocrystalline metals, International Journal of Solids and Structures, 45 (2008) 3897-3907.

[4] F. Yang, W. Yang, Crack growth versus blunting in nanocrystalline metals with extremely small grain size, Journal of the Mechanics and Physics of Solids, 57 (2009) 305-324.

[5] M.J. Buehler, F.F. Abraham, H. Gao, Hyperelasticity governs dynamic fracture at a critical length scale, Nature, 426 (2003) 141-146.

[6] S. Hai, E.B. Tadmor, Deformation twinning at aluminum crack tips, Acta Materialia, 51 (2003) 117-131.

[7] M.D.W.A.C.D. Farkas, Multiple-dislocation emission from the crack tip in the ductile fracture of Al, Philosophical Magazine A, 81 (2001) 1241-1255.

[8] D.H. Warner, W.A. Curtin, S. Qu, Rate dependence of crack-tip processes predicts twinning trends in f.c.c. metals, Nature materials, 6 (2007) 876-881.

[9] M. Wen, X.-J. Xu, Y. Omura, S. Fukuyama, K. Yokogawa, Modeling of hydrogen embrittlement in single crystal Ni, Computational Materials Science, 30 (2004) 202-211.

[10] D. Farkas, H. Van Swygenhoven, P. Derlet, Intergranular fracture in nanocrystalline metals, Physical Review B, 66 (2002).

[11] D. Farkas, S. Van Petegem, P.M. Derlet, H. Van Swygenhoven, Dislocation activity and nano-void formation near crack tips in nanocrystalline Ni, Acta Materialia, 53 (2005) 3115-3123.

[12] D. Farkas, S. Mohanty, J. Monk, Linear Grain Growth Kinetics and Rotation in Nanocrystalline Ni, Physical Review Letters, 98 (2007) 165502.

[13] D. Farkas, B. Hyde, Improving the ductility of nanocrystalline bcc metals, Nano Letters, 5 (2005) 2403-2407. 
[14] A. Latapie, D. Farkas, Molecular dynamics simulations of stress-induced phase transformations and grain nucleation at crack tips in Fe, Modelling and Simulation in Materials Science and Engineering, 11 (2003) 745-753.

[15] A. Latapie, D. Farkas, Molecular dynamics investigation of the fracture behavior of nanocrystalline $\alpha$-Fe, Physical Review B, 69 (2004).

[16] I.A. Ovid'ko, A.G. Sheinerman, Ductile vs. brittle behavior of pre-cracked nanocrystalline and ultrafine-grained materials, Acta Materialia, 58 (2010) 5286-5294.

[17] D. Sen, C. Thaulow, S.V. Schieffer, A. Cohen, M.J. Buehler, Atomistic Study of Crack-Tip Cleavage to Dislocation Emission Transition in Silicon Single Crystals, Physical Review Letters, 104 (2010).

[18] Y.-F. Guo, C.-Y. Wang, D.-L. Zhao, Atomistic simulation of crack cleavage and blunting in bcc-Fe, Materials Science and Engineering: A, 349 (2003) 29-35.

[19] V. Pelikán, P. Hora, A. MacHová, A. Spielmannová, Brittle-ductile behavior in 3D iron crystals, Czechoslovak Journal of Physics, 55 (2005) 1245-1260.

[20] Y. Mishin, M.J. Mehl, D.A. Papaconstantopoulos, A.F. Voter, J.D. Kress, Structural stability and lattice defects in copper: Ab initio, tight-binding, and embedded-atom calculations, Physical Review B - Condensed Matter and Materials Physics, 63 (2001) 2241061-22410616.

[21] M. Parrinello, A. Rahman, Polymorphic transitions in single crystals: A new molecular dynamics method, Journal of Applied Physics, 52 (1981) 7182-7190.

[22] J.D. Honeycutt, H.C. Andersen, Molecular dynamics study of melting and freezing of small Lennard-Jones clusters, Journal of Physical Chemistry, 91 (1987) 4950-4963.

[23] G.E. Beltz, D.M. Lipkin, L.L. Fischer, Role of crack blunting in ductile versus brittle response of crystalline materials, Physical Review Letters, 82 (1999) 4468-4471.

[24] I.A. Ovid'Ko, A.G. Sheinerman, Ductile vs. brittle behavior of pre-cracked nanocrystalline and ultrafine-grained materials, Acta Materialia, 58 (2010) 5286-5294.

[25] H. Van Swygenhoven, P.M. Derlet, A.G. Froseth, Stacking fault energies and slip in nanocrystalline metals, Nature materials, 3 (2004) 399-403.

[26] V. Yamakov, D. Wolf, S.R. Phillpot, H. Gleiter, Deformation twinning in nanocrystalline Al by molecular-dynamics simulation, Acta Materialia, 50 (2002) 5005-5020.

[27] J. Schiøtz, F.D. Di Tolla, K.W. Jacobsen, Softening of nanocrystalline metals at very small grain sizes, Nature, 391 (1998) 561-563.

[28] I.A. Ovid'ko, A.G. Sheinerman, E.C. Aifantis, Effect of cooperative grain boundary sliding and migration on crack growth in nanocrystalline solids, Acta Materialia, 59 (2011) 5023-5031.

[29] R.J. Nedoushan, M. Farzin, Effect of hydrostatic pressure on nano crystalline materials behavior, Journal of Nano Research, 18-19 (2012) 27-42.

[30] H. Li, F. Ebrahimi, Ductile-to-brittle transition in nanocrystalline metals, Advanced Materials, 17 (2005) 1969-1972.

[31] M. Tanaka, K. Higashida, T. Shimokawa, T. Morikawa, Brittle-ductile transition in low carbon steel deformed by the accumulative roll bonding process, Materials Transactions, 50 (2009) 56-63. 
Journal of Nano Research Vol. 23

10.4028/www.scientific.net/JNanoR.23

A Molecular Dynamics Simulation of Fracture in Nanocrystalline Copper

10.4028/www.scientific.net/JNanoR.23.50 